\title{
Correction to: First RILEM International Conference on Concrete and Digital Fabrication - Digital Concrete 2018
}

Timothy Wangler and Robert J. Flatt

\section{Correction to: \\ T. Wangler and R. J. Flatt (Eds.): \\ First RILEM International Conference on Concrete and Digital Fabrication - Digital Concrete 2018, RILEM Bookseries 19, https://doi.org/10.1007/978-3-319-99519-9}

The original version of the book was inadvertently published without incorporating the corrections, which were now updated as follows:

In Chapter "Experience in Online Modification of Rheology and Strength Acquisition of 3D Printable Mortars",

- In Page 25, Introduction, the sentence beginning with "Based on published information..." is deleted.

- In Page 26, Materials and Methods, the sentence beginning with "A similarly designed product..." is deleted.

- In Page 26, Materials and Methods, the sentence beginning with "This product has not yet been..." is deleted.

- In Page 34, Results, the sentence beginning with "And in the case of the NAG3..." is deleted.

- In Page 37, References, 9th and 10th references are deleted.

In Chapter "Challenges of Real-Scale Production with Smart Dynamic Casting", lowresolution Figure 4 is replaced with high resolution, Figure 5 is replaced with new figure and Figure 6 and the graph near are positioned as per the standard.

The updated online version of these chapters can be found at https://doi.org/10.1007/978-3-319-99519-9_3

https://doi.org/10.1007/978-3-319-99519-9_28

(C) RILEM 2019

T. Wangler and R. J. Flatt (Eds.): DC 2018, RILEM Bookseries 19, p. E1, 2019.

https://doi.org/10.1007/978-3-319-99519-9_31 